\title{
Surveillance for low-risk kidney cancer: a narrative review of contemporary worldwide practices
}

\author{
Helen Wei Cui, Mark Edward Sullivan
}

Urology Department, Oxford University Hospitals NHS Foundation Trust, Oxford, UK

Contributions: (I) Conception and design: None; (II) Administrative support: None; (III) Provision of study materials or patients: None; (IV) Collection and assembly of data: HW Cui; (V) Data analysis and interpretation: All authors; (VI) Manuscript writing: All authors; (VII) Final approval of manuscript: All authors.

Correspondence to: Helen Cui. Urology Department, Churchill Hospital, Old Road, Oxford, OX3 7LE, UK. Email: helen.cui@nhs.net.

\begin{abstract}
The management trend of low-risk kidney cancer over the last decade has been from treatment with radical nephrectomy, to use of nephron sparing procedures of partial nephrectomy and ablation, as well as the option of active surveillance (AS). This narrative review aims to summarise the available guidelines related to AS and review the published descriptions of regional practices on the management of low-risk kidney cancer worldwide. A search of PubMed, Google Scholar and Cochrane Library databases for studies published 2010 to June 2020 identified 15 studies, performed between 2000 and 2019, which investigated 13 different cohorts of low-risk kidney cancer patients on AS. Although international guidelines show a level of agreement in their recommendation on how AS is conducted, in terms of patient selection, surveillance strategy and triggers for intervention, cohort studies show distinct differences in worldwide practice of AS. Prospective studies showed general agreement in their predefined selection criteria for entry into AS. Retrospective studies showed that patients who were older, with greater comorbidities, worse performance status and smaller tumours were more likely to be managed with AS. The rate of percutaneous renal mass biopsy varied between studies from $2 \%$ to $56 \%$. The surveillance protocol was different across all studies in terms of recommended modality and frequency of imaging. Of the 6 studies which had set indications for intervention, these were broadly in agreement. Despite clear criteria for intervention, patient or surgeon preference was still the reason in 11-71\% of cases of delayed intervention across 5 studies. This review shows that AS is being applied in a variety of centres worldwide and that key areas of patient selection criteria and surveillance strategy have large similarities. However, the rate of renal mass biopsy and of delayed intervention varies significantly between studies, suggesting the process of diagnosing malignant SRM and decision making whilst on AS are varying in practice. Further research is needed on the diagnosis and characterisation of incidentally found small renal masses (SRM), using imaging and histology, and the natural history of these SRM in order to develop evidence-based active surveillance protocols.
\end{abstract}

Keywords: Kidney neoplasms; small renal mass; active surveillance; guidelines; practice

Submitted Sep 25, 2020. Accepted for publication Feb 04, 2021.

doi: $10.21037 /$ tau-20-1295

View this article at: http://dx.doi.org/10.21037/tau-20-1295

\section{Introduction}

Low-risk kidney cancer, in the context of this review, is defined as either: clinical stage T1a renal cell carcinoma with a localised renal tumour $\leq 4 \mathrm{~cm}$, or a kidney tumour suspected to be malignant based on imaging, of size $\leq 4 \mathrm{~cm}$ (also referred to as a small renal mass or SRM), or a Bosniak III or IV complex cyst (1).

The understanding of the natural history, prognosis 
and treatment outcomes for low-risk kidney cancer has improved with the use of retrospective and more recently, large, prospective observational studies, leading to recent changes in the guidelines on the management of these tumours. The most significant change in management has been the shift in emphasis to partial nephrectomy $(\mathrm{PN})$ as the recommended surgical treatment for SRM in place of radical nephrectomy ( $\mathrm{RN})$, based on the evidence for non-inferior oncological outcomes and the potential benefit of preserving nephrons $(2,3)$. In addition to the increased uptake of $\mathrm{PN}$, there has been increasing interest in surveillance as an active management strategy for small renal masses, given the evidence for their natural history to be indolent, slow growing and of limited metastatic potential (4). Surveillance may also be of greater benefit to more elderly and comorbid patients who have competing risks for mortality, for which operative primary intervention (PI) may be considered overtreatment (5). Given the prominence of AS in the management of the SRM and the lack of consensus on the standards for AS, this review aims to summarise the most recent international guidelines pertaining to AS, and to compare the practice of AS worldwide within the last 2 decades by comparing studies with AS cohorts. The specific areas compared were the selection criteria and decision-making processes for choosing AS compared to surgical or ablative treatment, the role of renal mass biopsy (RMB), the strategy employed for surveillance and the outcomes of AS in terms of delayed intervention (DI) and survival.

\section{Search strategy}

Understanding of contemporary worldwide practice was gained from a search of the guidelines from worldwide urological associations as well as a literature search for studies documenting AS cohorts. Urological associations listed on the Urological Worldwide Society Database (accessed from www.auanet.org/education/internationalsocieties) were searched for whether they had a published guideline accessible online. If a published guideline or recommendation from a regional urological association could be found, this was included as the example of practice for that region.

A literature search of studies published in English, with full text available, in the period 2010 to June 2020 which provide evidence for contemporary practice was conducted. We searched the PubMed, Google Scholar and the Cochrane Library databases using the following medical subject heading terms 'kidney neoplasms', 'localised' or 'small renal mass', and 'practice', 'with or without delayed intervention' to identify all full text articles, in English, reporting on the observation of suspected low-risk renal cancers, with study periods from 2000 onwards. Cited references were searched and retrieved for potentially eligible publications containing cohorts of AS patients. All paediatric renal cancer, and studies which included greater than $\mathrm{cT} 1(>7 \mathrm{~cm})$ tumours, were excluded. Study titles and abstracts were reviewed to identify all series that analysed management of localised renal tumours and discussed their natural history or outcomes of surveillance, with, or without comparison to other treatment. The following data was extracted from the studies: definition of low-risk kidney cancer, period of study, baseline and clinical characteristics of study population, patient selection criteria, surveillance protocol, proportion of patients who had $\mathrm{RMB}$, triggers for delayed intervention, and clinical outcomes during follow-up.

\section{Findings}

\section{Search results of the international guidelines}

The Urological Worldwide Society Database listed 98 urological bodies (6). Separate European and USA organisations were not counted as these were represented by the European Association of Urology (EAU) and American Urological Society (AUA). Online published guidelines were available for 8 organisations: EAU (7), AUA (8), European Society for Medical Oncology (ESMO) (9), Canadian Urological Association (CUA) (10), Japanese Urological Association (JUA) (11), Taiwan Urological Association, Confederacion Americana de Urologia (CAU), Argentinian Urological Society (12) and the Saudi Urological Association (13). The guidelines for the region of Taiwan refers to the EAU guidelines, and the urological societies of the countries of South America represented by the CAU was the same as the AUA guidelines. Further recommendations for the Latin American Renal Cancer Group were found during the literature search (14). Therefore, 7 sets of guidelines were reviewed and recommendations regarding active surveillance are summarised in Table 1.

\section{Summary of guidelines from urological associations}

Results of the guidelines available on recommendations for AS and the worldwide practice of management of SRM and AS show many similarities, although this is skewed towards 

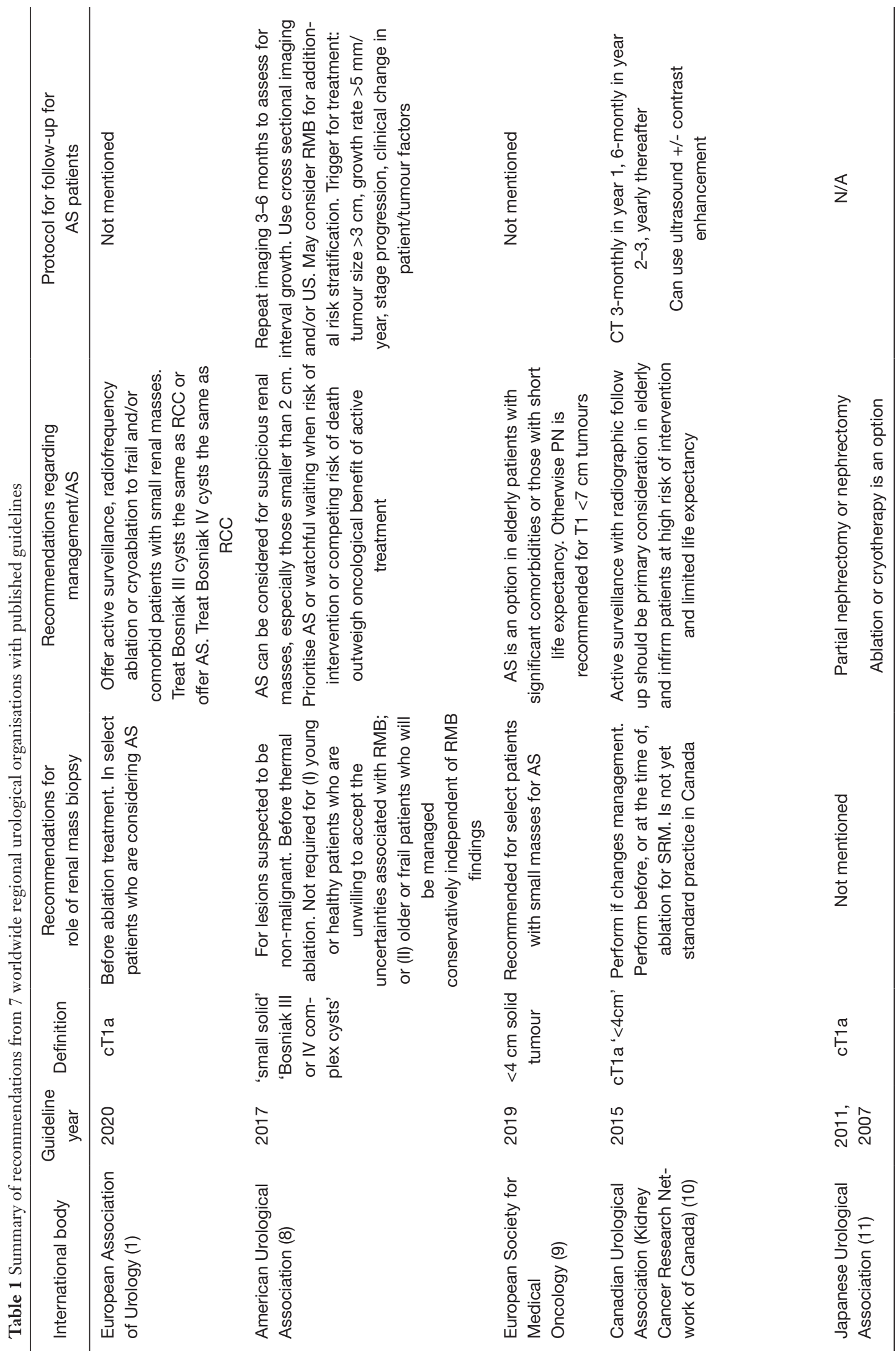

ำ
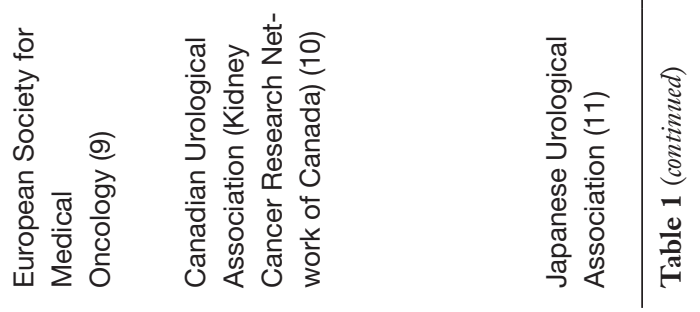


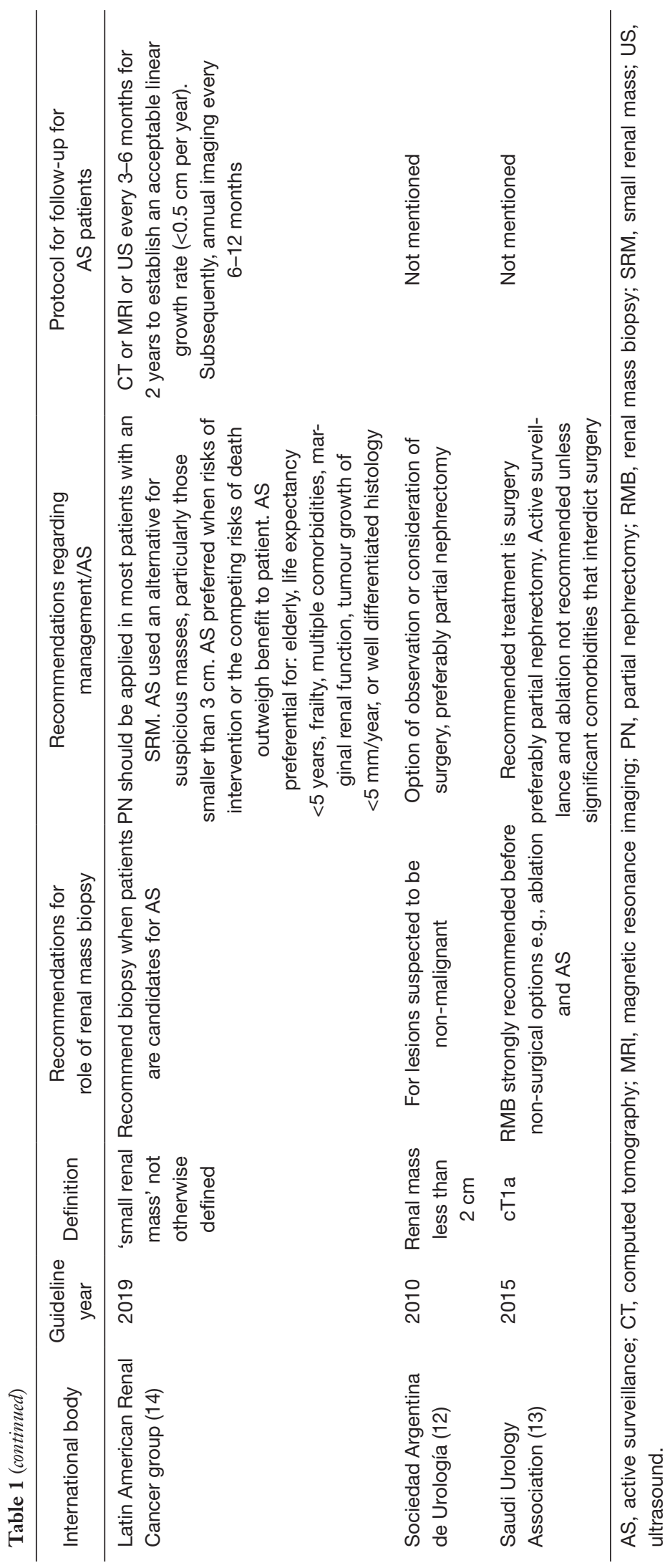


guidelines and practice pertaining to North America and Europe.

The EAU, ESMO and CUA relate to c T1a renal tumours, whereas the AUA emphasises a recommendation for AS in renal tumours less than $2 \mathrm{~cm}$. In terms of patient selection, the EAU, AUA, ESMO and Latin American Renal Cancer group guidelines recommend AS for comorbid patients and elderly patients, with the rationale that primary intervention is likely to outweigh oncological benefit. No AS imaging protocol is specified in the EAU or ESMO guidelines, whereas the AUA, CUA and Latin American Renal Cancer group all have similar imaging protocols of more intensive imaging 3 to 6-monthly in the first year, with longer intervals thereafter. Only the AUA guidelines provide more specific definitions for progression as a trigger to treatment (more than $5 \mathrm{~mm}$ growth per year or to more than $3 \mathrm{~cm}$ or change in clinical stage or patient factors).

The recommendation for RMB vary between guidelines. The EAU, AUA, CUA and Saudi Urology Association guidelines recommend RMB before ablation treatment for SRM. For patients being considered for AS, RMB is recommended by the Latin American Renal Cancer group, and strongly recommended by the Saudi Urology Association guidelines, and recommended for select AS patients in the EAU and ESMO guidelines. The AUA and Argentinian Society of Urology guidelines also recommend RMB for suspected non-malignant lesions.

\section{Search results of studies pertaining to worldwide practice}

We identified 181 unique citations; of these 144 were excluded after review of the abstract due to reasons of: not containing the population of interest, study period starting before 2000, review articles, commentaries or studies without relevant clinical data on both patient characteristics and outcomes. Full text screening was carried out in 37 articles, of which 21 were excluded, resulting in 16 included articles (of which 2 are conference abstracts) covering 13 different patient cohorts represented in 15 studies (15-30). Numerous studies have been published using the large United States datasets of DISSRM (Delayed Intervention and Surveillance for Small Renal Masses registry) (31-36), SEER (Surveillance, Epidemiology and End Results program) (37-41) and the National Cancer Database $(42,43)$, of which, the most relevant articles for this review have been chosen. Regions represented were North America, United Kingdom, Israel, and South Korea. The DISSRM study data is represented twice in Table 2 to distinguish data available from the full text article published in 2015 (20) and the updated data published in a 2020 abstract (21). The Oxford study combines data from the 2012 published full text article (which contained an AS cohort of 71 patients only) (23) and the 2020 updated abstract (which contained a cohort of 208 patients with SRM undergoing AS, PN or RN) (24).

\section{Studies pertaining to worldwide practice}

Fifteen studies covering 13 different datasets are summarised in Table 2, of which the vast majority relate to North American practice. The University of Michigan published two separate studies using the same SRM database $(15,26)$. The earlier study included all SRM and the later study included patients on AS only. Two articles relate to the DISSRM registry, one published in 2015 (20) and an updated abstract published in 2020 (21). The two studies published from Canada may represent some overlap of patient samples as some centres were used in both studies. The University of Toronto study (19) included 82 patients with cT1 renal masses from 3 centres, whereas the RCC Consortium study included 178 patients with cT1a renal masses from 8 centres (22). Three studies [University of Michigan 2012 (15), Fox Chase Cancer Center (16), National Cancer Database (NCDB) (17)] looked at population trends and characteristics of patients on AS with no follow up data. Of the other 11 studies with follow up data, 7 studies [University of Toronto (19), Canada Renal Cell Carcinoma (RCC) Consortium (22), Haifa (25), University of Michigan 2016 (26), Pusan National University (27), Tayside (28), MD Anderson (30)] included AS patients only, and the remaining studies [Cleveland Clinic (18), DISSRM (20,21), Oxford (23), SEER (29)] included patients on SRM who underwent a variety of treatments including RN, PN and ablation. Most of the studies are retrospective analyses of prospectively entered databases of SRM or AS patients, with two studies of population databases using the SEER Medicare linked database (29) and the NCDB (17), both from USA. The only prospectively recruited clinical trial, which was non-comparative, was the Canada RCC Consortium study (22). Other prospectively enrolled AS cohorts were from the University of Toronto (19), Pusan National University (27), Tayside Urological Cancers Network (28), Texas MD Anderson Cancer Center (30) and the DISSRM registry $(20,21)$.

\section{Trends in the management of SRM}

Evidence of management trends of SRM over the last 2 decades can be gained from analysis of patient databases from the United States. The NCDB study (17) showed that, 


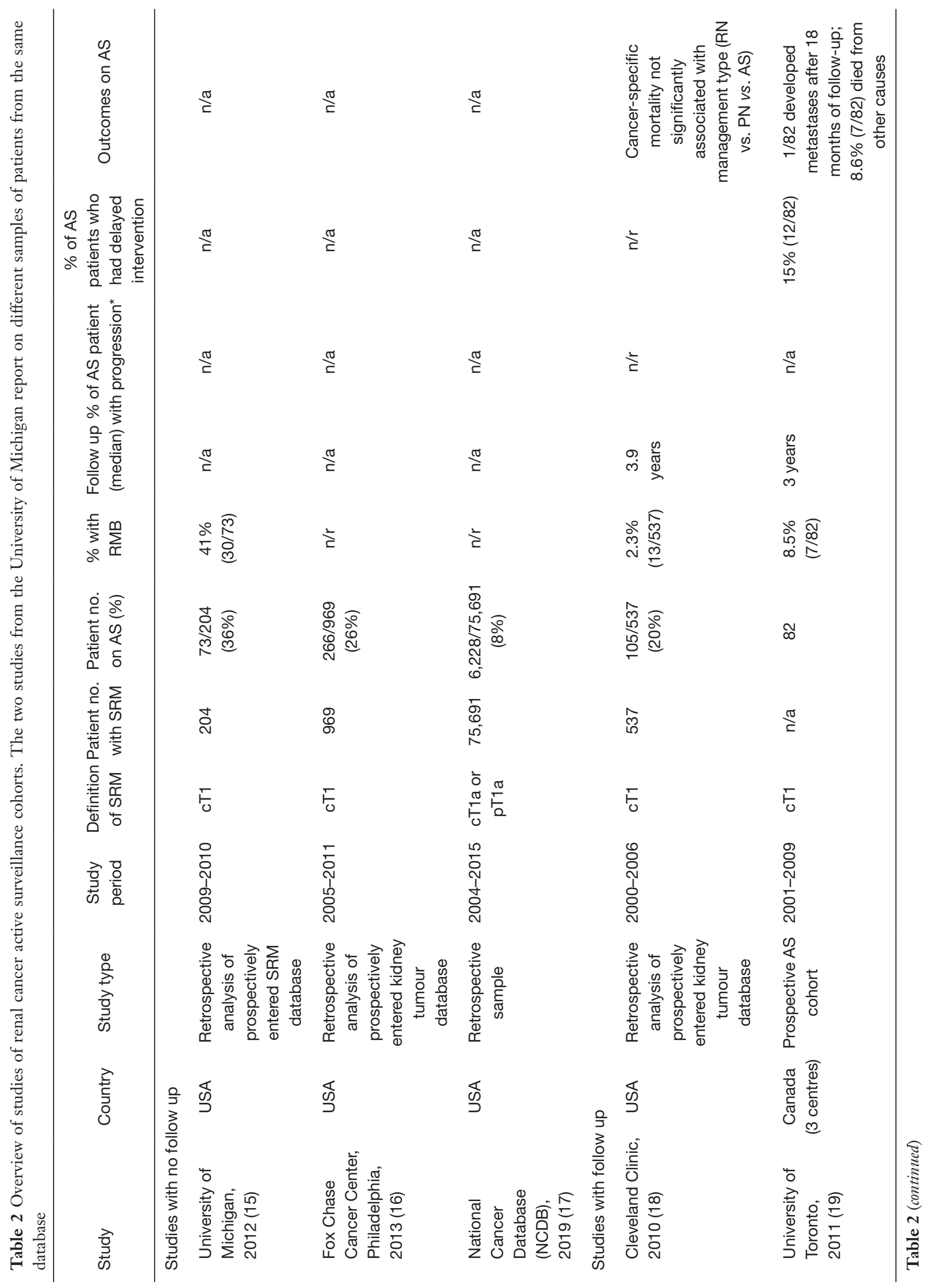




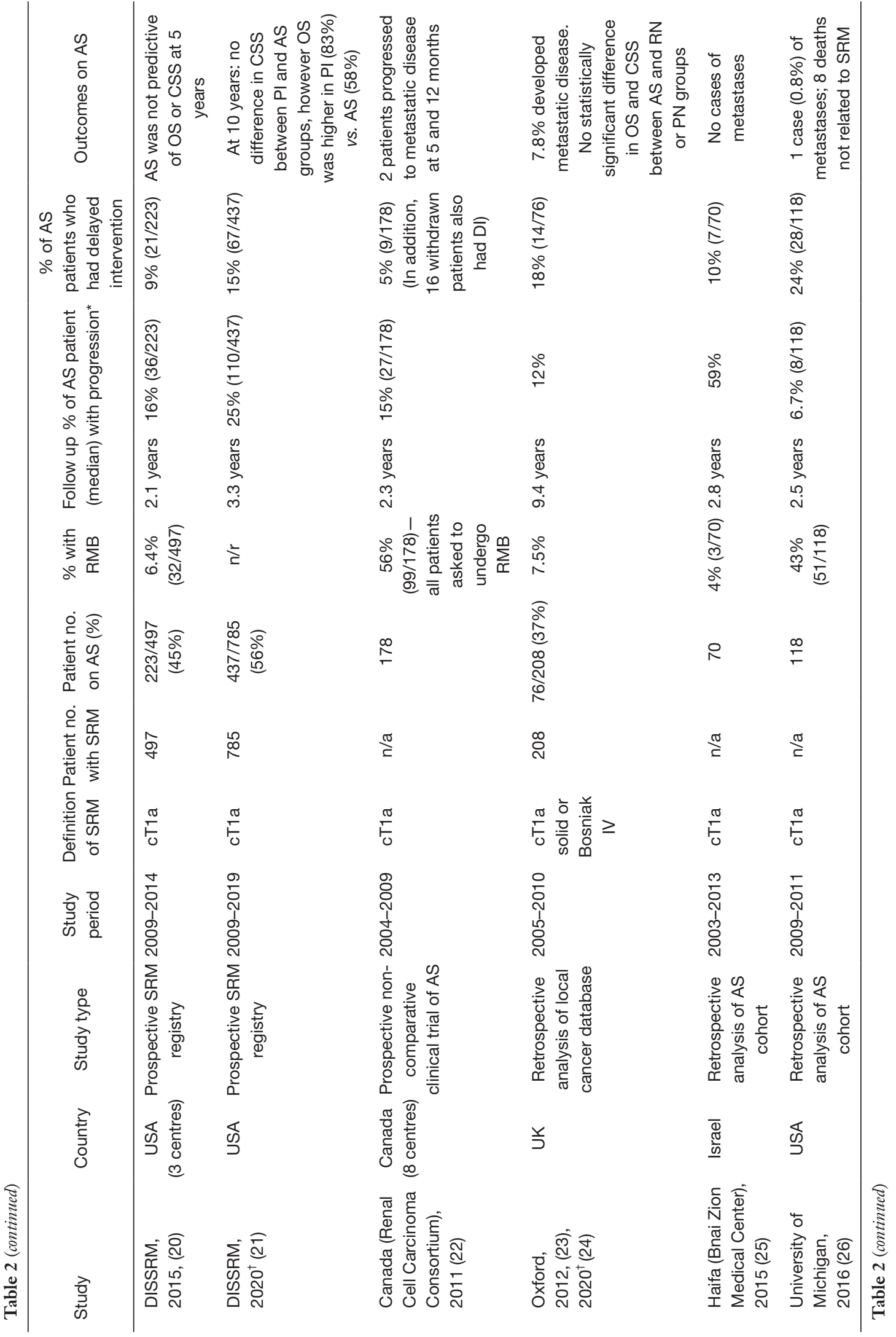




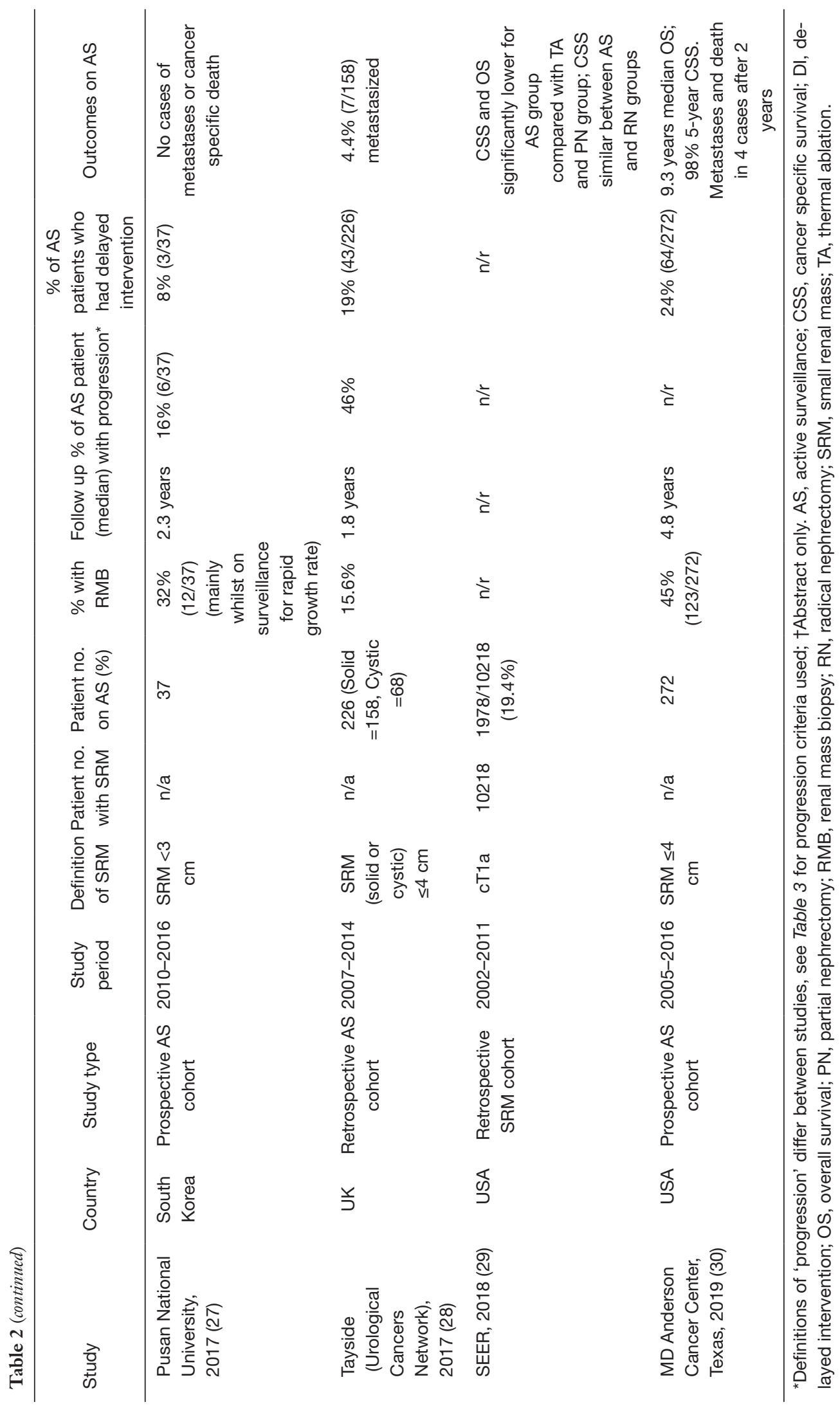




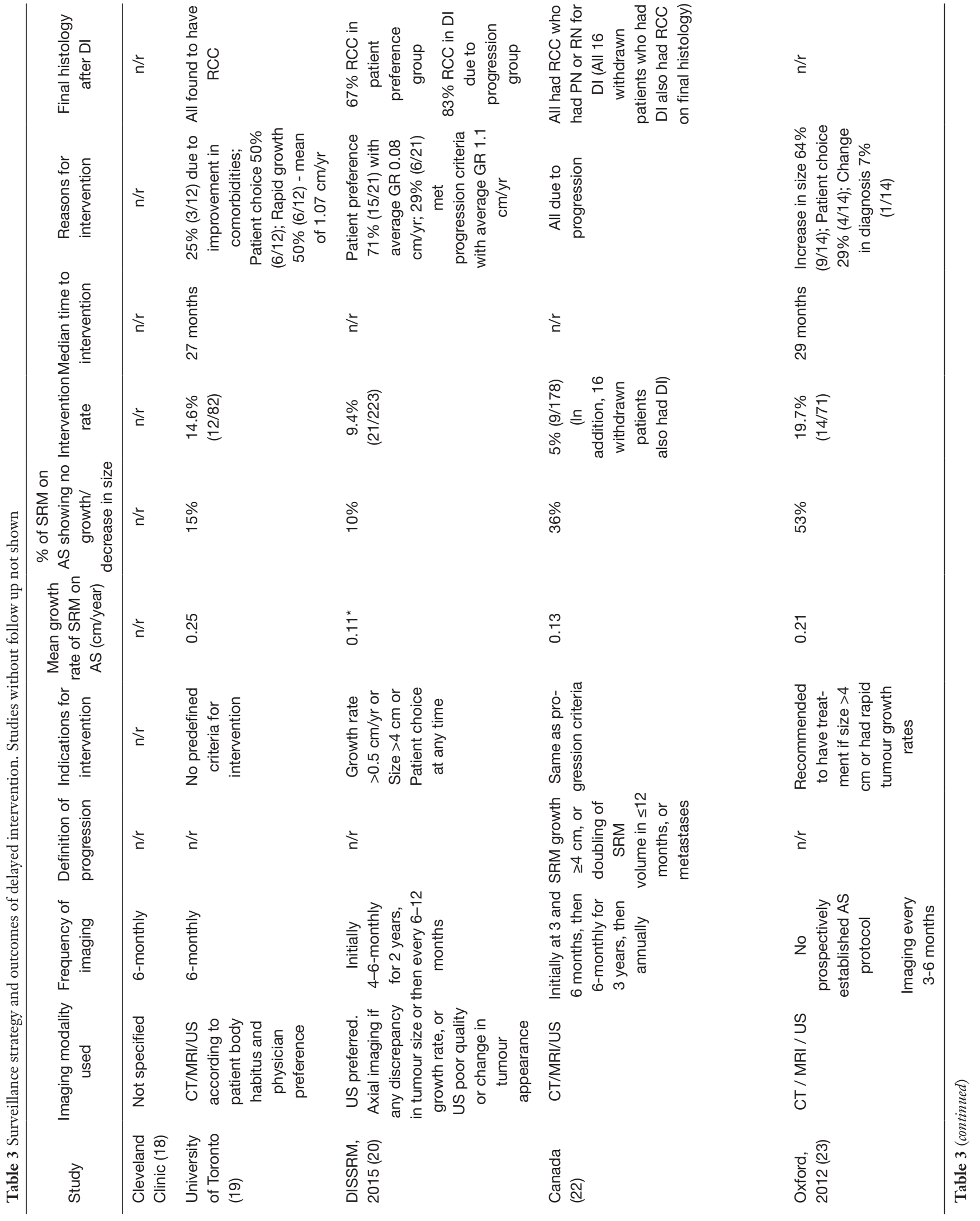




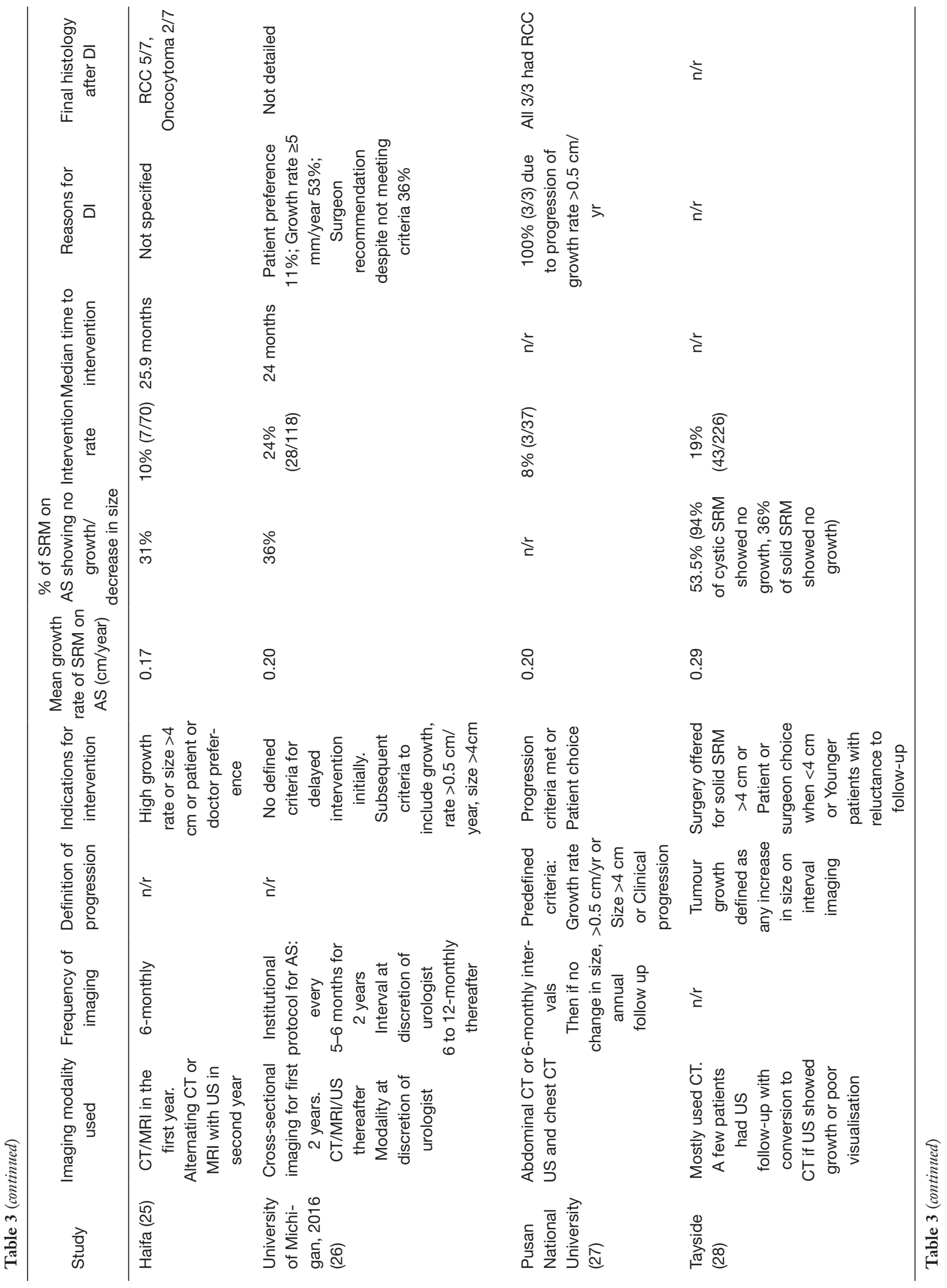




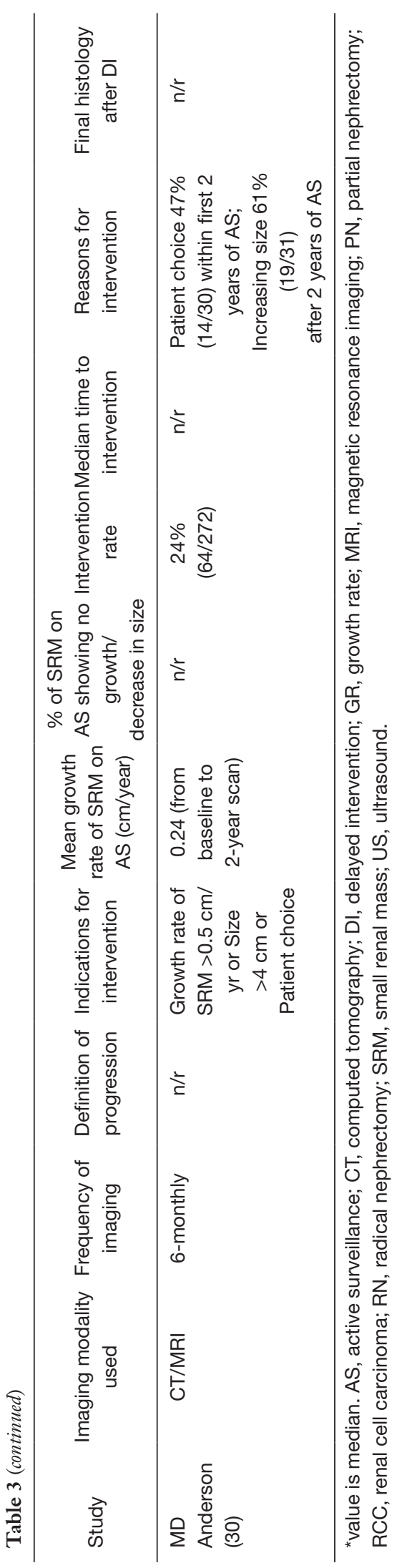

between 2004 and 2015, of the 75,691 patients found with cT1a renal mass, $8 \%$ were managed with AS. The SEER study (29) showed that $19.4 \%$ of the 10,218 patients with cT1 a renal mass were managed with AS.

The main findings from analysis of several studies using the NCDB and SEER data is that surgery is the mainstay of treatment for stage 1 kidney tumours, and PN uptake has increased dramatically in recent years in all age groups, except in the 90+ age group, with lower rates of increase in use of ablation and largely static uptake of AS, even in older age groups $(17,39,42,44)$.

Analysis of recorded renal cancers NCDB for the period 1993 to 2007 showed the use of PN for stage 1 renal tumours increased from $6.3 \%$ to $32 \%$ (44), with a further increase in use of PN for T1a tumours to 57\% by 2015 (17), with similar trends found from analysis of the SEER registry from 1998-2008 (39). Conversely, the use of AS for T1a tumours was stable between 2004 (7.3\%) to 2015 (8.2\%) across all age groups (17). Over this period, approximately $10 \%$ of $70-79$-year olds, and $30 \%$ of $80-89$ year olds were managed with AS with no significant change in trend in use of AS over these years, in contrast to increasing rates of $\mathrm{PN}$ and ablation (17). This lag in growth of AS compared to $\mathrm{PN}$ and ablation in all age groups suggests that urologists were continuing to opt for primary intervention for SRM, and this may be resulting in an overtreatment of the SRM for those suitable for AS $(17,45)$. This may also have been influenced by the increased uptake of robotic partial nephrectomy which has outpaced the adoption of AS for SRM (45).

\section{Patient selection into AS Selection criteria for $A S$}

An overview of important patient characteristics and selection criteria is detailed in Table 4. Only one DISSRM publication from 2015 is included in Table 4 to illustrate patient selection criteria for this study. The SEER study is not included as it contained no information on patient selection for AS. Information on how patients were selected for AS varied between studies depending on whether they included retrospective analyses of patient cohorts, or if the study had specific inclusion and exclusion criteria for enrolment into an AS protocol. Three prospective AS cohort studies [Canada (22), University of Toronto (19), MD Anderson (30)] had specific criteria or recommendations for eligibility for AS which were broadly in agreement. The selection criteria for patients to be managed with AS was most clearly defined in the prospective clinical trial from 


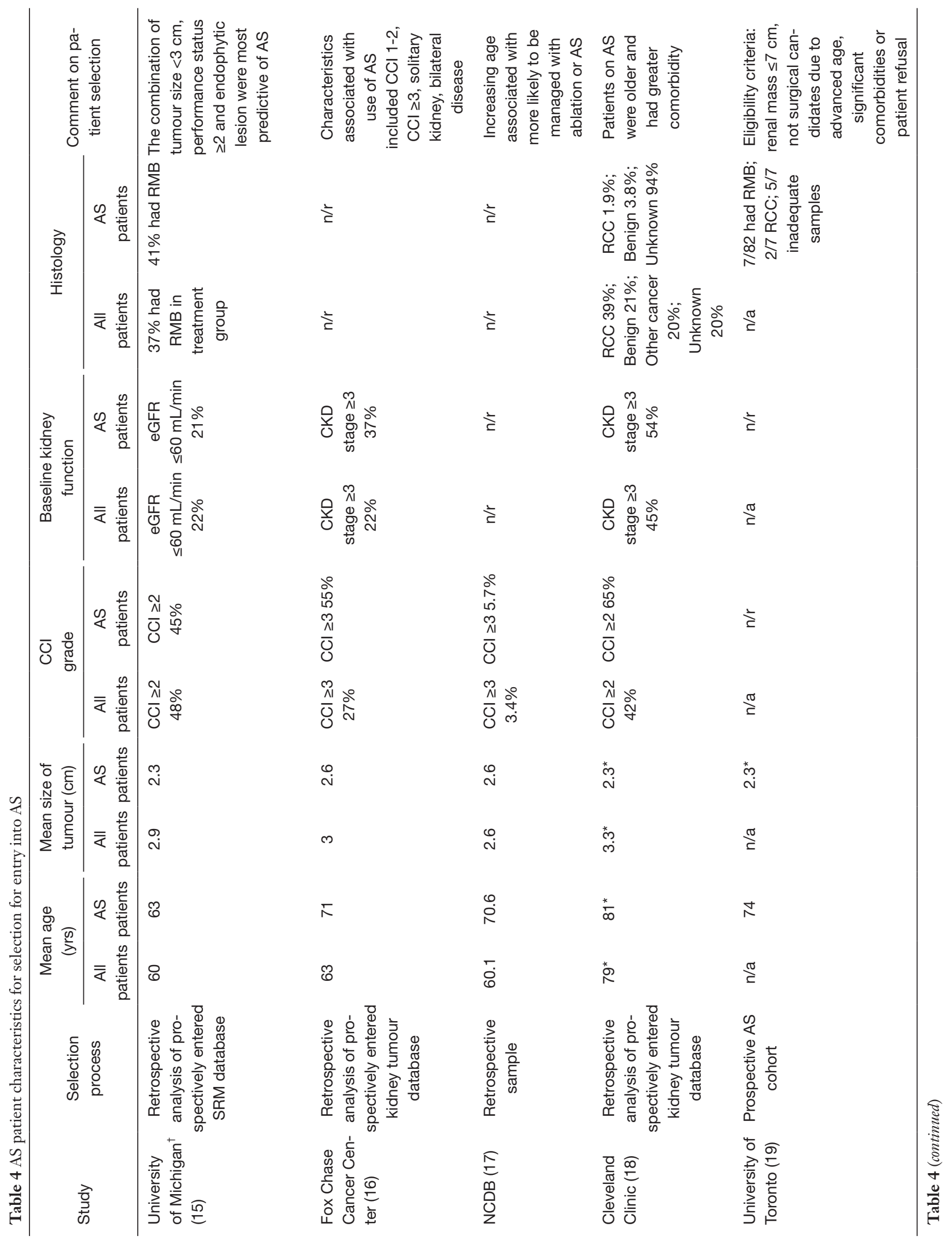




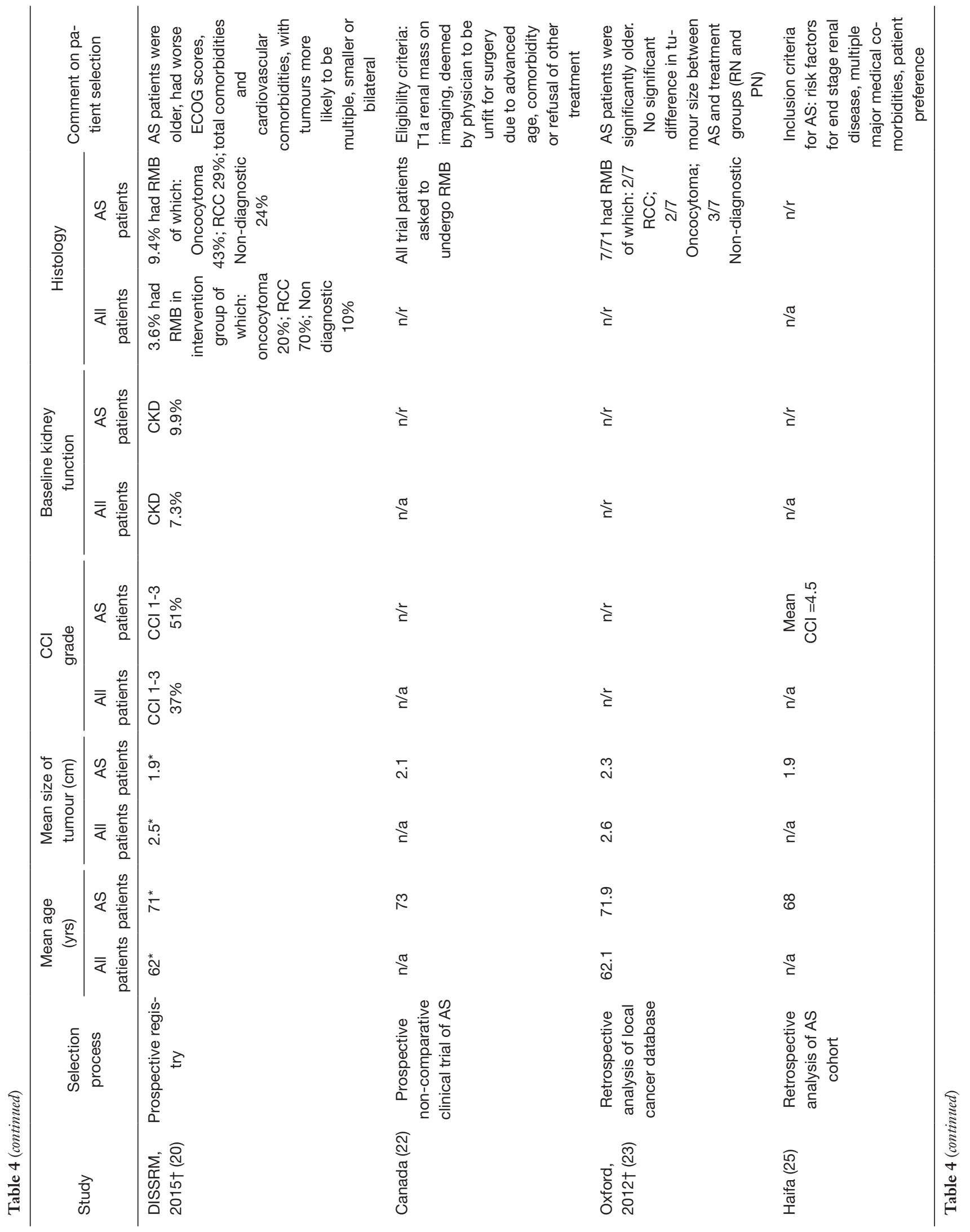




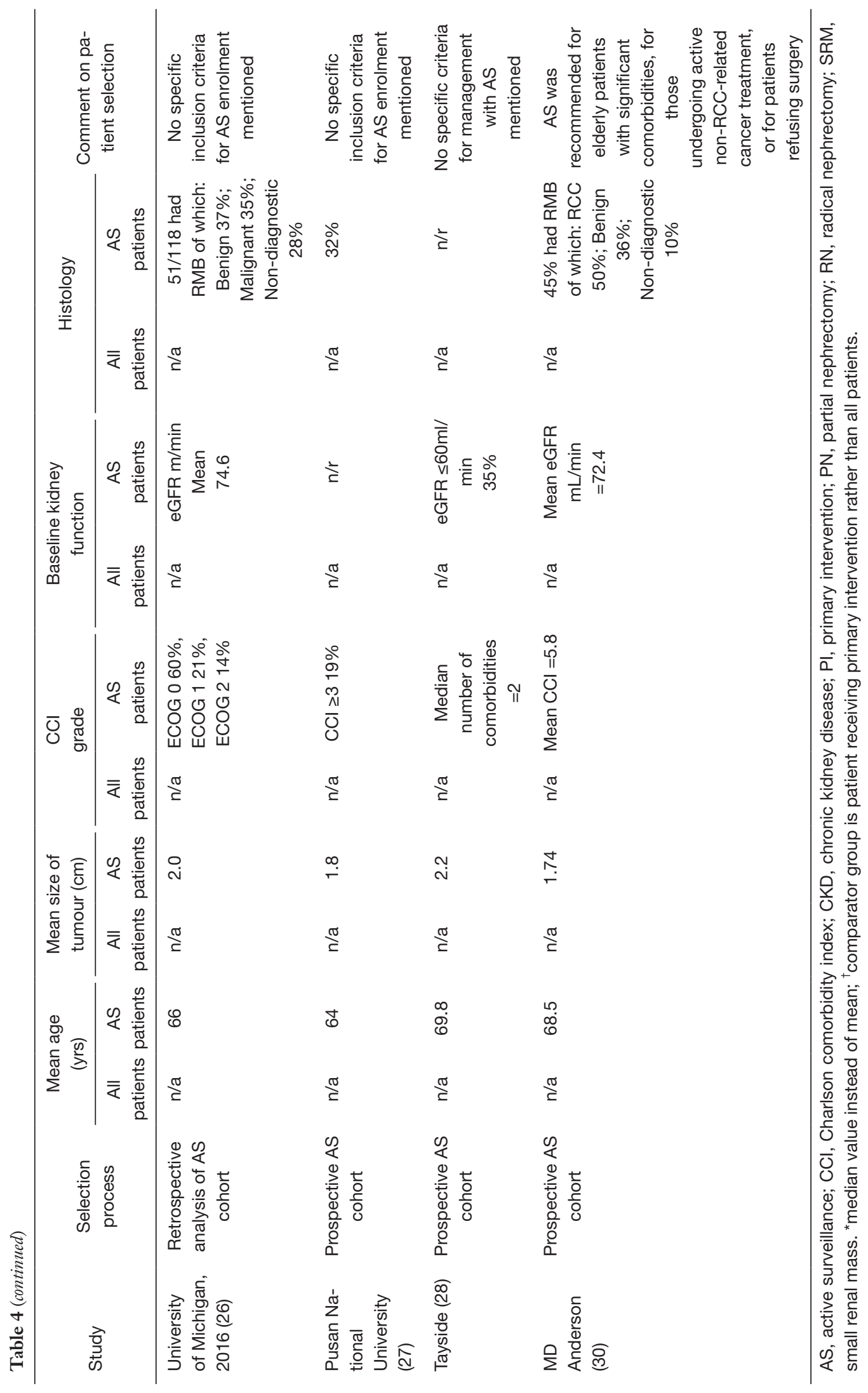


the Canada RCC consortium study (22). Eligibility criteria included a T1aN0M0 renal mass on imaging, and the patient to have been deemed by their responsible physician to be unfit for surgery due to advanced age, comorbidity, or refusal of other treatment. Patients were ineligible if they had an estimated life expectancy of less than 2 years, had been diagnosed with a SRM more than 12 months before or were on concurrent systemic therapy for other malignancies. Each participant was also asked to undergo $\mathrm{RMB}$, resulting in the study with the highest proportion of AS patients who have had RMB, although this was still only $56 \%$.

The University of Toronto study (19) was a prospectively accrued cohort of AS patients which also had predefined selection criteria. The inclusion criteria for enrolment on this AS study was a renal mass $\leq 7 \mathrm{~cm}$ and that patients were deemed not to be surgical candidates because of advanced age, significant comorbidities, or patient refusal. RMB was not a requirement for inclusion and only $8.5 \%$ of patients had RMB.

The MD Anderson study (30) was a prospective AS cohort which did not have strict eligibility criteria but stated that AS was recommended for elderly patients with significant comorbidities, or undergoing non-RCC related active cancer treatment, or for patients refusing surgery. Although the study from Haifa (25) was a retrospective analysis of AS patients, the institution had existing criteria for patient selection for AS which was: contrast enhancing renal mass of $4 \mathrm{~cm}$ or less, with risk factors for end-stage renal disease, multiple major comorbidities, and patient preference.

The other 3 studies of prospective AS cohorts [DISSRM (20), Pusan National University (27), Tayside (28)] were not as prescriptive in their inclusion criteria. Patients were eligible based on size of renal mass, and the availability of cross-sectional imaging. The DISSRM registry specified a solid enhancing renal mass $\leq 4 \mathrm{~cm}$, whereas the Tayside cohort included both solid and cystic SRM $\leq 4 \mathrm{~cm}$. The DISSRM registry (20) also specified exclusion criteria of patients with a personal history of RCC, familial RCC syndrome or suspicion of second malignancy metastatic to the kidney. In summary, these selection criteria appear to show an emphasis on patient characteristics and ineligibility for surgery rather than the risk profile of the tumour.

\section{Clinical characteristics of patients on AS}

Six studies included statistical analysis of comparison of the baseline characteristics between the AS group and either all SRM patients or a primary intervention (PI) group. Five of these studies were retrospective analyses of datasets of SRM patients [University of Michigan 2012 (15), Fox Chase Cancer Center (16), NCDB (17), Cleveland Clinic (18), Oxford (23)] and one study, the DISSRM registry (20), used prospective enrolment.

The patients selected for AS appear to be more likely to have a greater number of comorbidities and a decreased performance status. However, the significance of older age, renal failure and smaller tumour size in the AS groups varied across the studies. The Fox Chase Cancer Center study (16) examined the clinical characteristics of cT1 renal masses for selection of treatment. Out of a total of 969 patients, $25.7 \%$ were managed with AS and these patients were found to be more likely to have Charlson Comorbidity Index (CCI) score of 1 to 2 ( $\mathrm{OR}=2.2)$ or $\mathrm{CCI} \geq 3$ ( $\mathrm{OR}=6.9)$, have a solitary kidney $(\mathrm{OR}=7.1)$ and bilateral disease $(\mathrm{OR}=7.6)$. Patients with chronic kidney disease (CKD) stage $\mathrm{V}$ disease, history of previous malignancy, increasing tumour size, and high nephrometry score complexity were less likely to undergo expectant management with $\mathrm{RN}, \mathrm{PN}$ or ablation. Although the median age of those in the AS group was the highest at 71 years compared to 63 years in the $\mathrm{RN}$ group and 59 years in the $\mathrm{PN}$ group, there was no statistically significant increased odds of older age being selected for AS compared to $\mathrm{RN}(\mathrm{OR}=1.02)$.

The University of Michigan study (15) found the differences between the AS group and treatment group were: ECOG performance status, tumour size and whether the lesion was more than $50 \%$ exophytic. Patients with an ECOG score $<2$ were more likely to undergo treatment, and those with an ECOG score $\geq 2$ were more likely to undergo AS. There was no difference between the AS and treatment groups in clinical) characteristics of age, baseline renal function, CCI score, body mass index (BMI), or presence of a solitary kidney. A smaller tumour favoured AS, as did an entirely endophytic lesion on imaging.

The DISSRM prospective registry (20) showed a marked difference in characteristics of patient age, tumour size and comorbidities between patients on AS and those who had PI. The AS patients were older, had worse performance status, increased total comorbidities and cardiovascular comorbidities, and more likely to have multiple, smaller or bilateral tumours compared to patients who initially chose PI. There was no significant different between AS and PI groups for BMI, prior surgery, or tumour complexity. Fewer patients $(3.6 \%)$ in the PI group had RMB compared to the AS group (9.4\%).

In summary, the selection process or selection criteria for entry into AS showed similarities between studies. 
There were no studies that described objective measures for patient characteristics such as an age or CCI threshold. The description of the selection criteria recommendations suggests much is left to the discretion of the clinical team to decide on their judgement of 'advanced age' or 'significant comorbidities' or to be unsuitable for surgery. No studies had RMB as a prerequisite to being managed with AS. Some of the more technical considerations such as patient BMI and previous surgery have not been shown to be significant, whereas patient comorbidity and life expectancy, using CCI as a prognostic indicator, do appear to have been a significant consideration in decision-making between the patient and the clinician for selection into AS.

\section{Surveillance strategy and indications for delayed intervention}

Table 3 provides an overview of surveillance strategies used or predefined by the ten AS patient cohorts with follow-up included in this review. Although the SEER study (29) had follow-up for survival analysis, no information was available on surveillance strategy as this was a population study and therefore the SEER study is not included in Table 3. Six studies of prospective AS cohorts [University of Toronto (19), DISSRM (20), Canada (22), Haifa (25), Pusan National University (27), MD Anderson (30)] had a predefined study protocol or institutional protocol for AS surveillance. The Cleveland Clinic (18) study was retrospective and stated imaging was conducted 6-monthly, and the Oxford study had no institutional AS protocol, stating that imaging was usually every 3-6 months on surveillance. The Tayside study (28) also had no prospectively established protocol, with decisions made for SRM patients at multidisciplinary meetings (MDT), resulting in most patients receiving computed tomography (CT) surveillance.

\section{Modality and frequency of imaging surveillance}

All ten AS patient cohorts included imaging surveillance with clinical visits as part of the surveillance strategy, with general agreement between studies on the modality and frequency of imaging. After baseline cross-axial imaging, all studies (except for the Cleveland Clinic) described imaging with either CT, magnetic resonance imaging (MRI) or ultrasound scan (USS). The Haifa study (25) recommends alternating cross-sectional imaging with USS in the second year. Where USS is one of the modalities, care is taken to recommend cross-sectional imaging if the USS showed growth, change in the qualitative appearance of the tumour or provided poor quality images in the Tayside (28) and DISSRM studies (20).
Imaging frequency was broadly similar between studies, with a frequency of 3 to 6-monthly in the first 1 to 2 years, lengthening to 6-monthly or annually thereafter.

\section{Indications for delayed intervention}

Six studies had set criteria for progression or indications for intervention, of which five studies [DISSRM (20), Canada (22), Haifa (25), Pusan National University (27), MD Anderson (30)] had pre-defined criteria, and one study [University of Michigan (15)] introduced progression criteria during the period of the study. The Oxford (23) and Tayside (28) studies had recommendations for offering treatment rather than predefined criteria. The Cleveland Clinic (18) and University of Toronto (19) studies did not provide definitions of progression or indications for intervention.

The most commonly stated criteria for intervention, used in 4 studies [DISSRM, University of Michigan (15), Pusan National University (27), MD Anderson (30)], were growth of more than $0.5 \mathrm{~cm} /$ year or tumour size reaching $>4 \mathrm{~cm}$. Two studies [Oxford (23), Haifa (25)] included growth rate described as 'rapid' and 'high' rather than a defined rate. The Tayside study (28) did not include growth rate as a criterion. The Canada trial (22) used doubling of SRM volume in 12 months or less rather than a measure of maximal axial diameter for growth rate. Five studies [DISSRM (20), Haifa (25), Pusan National University (27), Tayside (28), MD Anderson (30)] include patient or surgeon choice as an indication, with only the Canada trial (22) not specifying this as a criteria. Although the Oxford study does not mention patient choice as an indication for treatment, $4 / 14$ patients who received DI were due to patient choice.

\section{Rate of delayed Intervention}

Nine studies [University of Toronto (19), DISSRM (20,21), Canada (22), Oxford (23), Haifa (25), University of Michigan (15), Tayside (28), Pusan National University (27), MD Anderson (30)] reported the rate of delayed intervention (DI) which varied between $5 \%$ to $24 \%$ and this is likely to be due to a variety of reasons The lowest DI rate of $5 \%$ in the Canada RCC Consortium study (22) may be due to the fact this was a trial with predefined criteria for intervention, and therefore patient preference was less likely to initiate DI. However, the true DI rate of the Canada study is higher, due to 16 patients who withdrew from the study also going onto to have treatment for their SRM. In total 33 out of the original 178 patients enrolled in the study withdrew due to patient or clinician preference. The cases of DI in the Haifa (25) and Pusan National University (27) studies were also all due to progression of the SRM. Five other studies [University 
of Toronto (19), DISSRM (20), Oxford (23), University of Michigan 2016 (15), MD Anderson (30)] stated patient preference as a reason for DI, with proportions of DI due to patient preference varying from $11 \%$ to $71 \%$. The $\mathrm{MD}$ Anderson study (30) also found that patient choice was the most common reason for DI within the first two years of AS, whereas increasing size was the most common reason after 2 years of AS. Increase in size or meeting progression criteria as the reason for DI varied from $29 \%$ to $100 \%$ of DI cases. All studies in Table 3 had a similar median follow up time of around 2-3 years, except for the Oxford study which had updated data at 9.4 years median follow up and the MD Anderson study which had a slightly longer median follow-up period of 4.8 years. There appears to be no relationship between length of follow-up and DI rate, to suggest that a longer follow up period will result in increasing numbers of patients receiving treatment. No firm conclusions can be drawn on the reasons behind the variation in DI rates across the studies but is likely to be due to the different selection criteria, time period covered by the study, surveillance strategies and criteria set for intervention as well as unmeasured factors of the regional differences in practice and preferences of the physicians.

\section{Discussion}

There have been several studies investigating the outcomes of AS for low-risk kidney cancer and multiple narrative $(5,47-50)$ and systematic reviews $(51,52)$ conducted on the clinical characteristics of patients with SRM and on AS, the natural history of SRM and the outcomes of AS. This review of contemporary practice of AS in the last 2 decades has found that most of the evidence regarding worldwide practice of AS is from North American cohorts and that large similarities can be found between studies in terms of patient selection and surveillance strategy for AS management. However, the practice of AS, as described in the studies included in this review, appears to rest on a handful of general principles, with plenty of room for interpretation left to the clinicians and also a strong emphasis on patient preference. More nuanced and evidence-based strategies for patient selection and surveillance have not been employed. Important information on the natural history of the SRM, the method of diagnosis and risk stratification of the SRM and the optimal surveillance strategy is still lacking to be able to aid informed decision making for choosing AS. The pertinent questions related to AS from diagnosis to decision for delayed intervention is discussed below.

\section{Patient and tumour selection for active surveillance}

\section{Competing risk of mortality from other causes}

Patient factors are increasingly relevant to consider when choosing a management option for SRM as the age group with the largest rate of increase of diagnosis of kidney cancer is 70-90 years (4). Significant patient factors identified from the studies in this review include age, comorbidities including concurrent other cancers, risk of morbidity and mortality from surgical intervention, and risk of worsening pre-existing renal failure or cardiovascular disease. Analysis of older patients (over 75 years) in population-based studies comparing the oncological outcomes of surgery (either PN or RN or ablation) to non-surgical management (either AS or watchful waiting), showed no significant benefit in cancer-specific mortality for surgical treatment (37).

In the elderly and those with significant comorbidities, there is the concept of 'competing risk' of other causes of mortality which are higher than the risk of renal cell cancerspecific mortality from the SRM $(1,18,50,53)$. A review of AS for SRM specifically in the elderly (aged $\geq 70$ years), showed an overall mortality of $15-51 \%$ across 17 retrospective studies, during up to 91 months of follow up on AS, with minimal $(0-5 \%)$ cancer-specific mortality or progression to metastases, regardless of type of primary intervention or on AS (50). Using the SEER database, modelling for mortality risk reveals that, in a 75 year old Caucasian male with a $4 \mathrm{~cm}$ renal tumour, the risk of mortality would be $5 \%$ from renal cancer, $4.5 \%$ from other cancer, and $14 \%$ from a non-cancer related cause (38).

\section{Age as a patient factor}

The guidelines and trends in management of SRM suggest that patient age is a major factor in the likelihood of being managed with AS. The DISSRM registry showed that $40 \%$ of patients initially chose AS, with the AS group being significantly older than the primary intervention group (median age 71 vs. 62 years old) (20). Although no age thresholds are mentioned in the international guidelines, the modelling suggests those over $70-75$ years receive less oncological benefit from primary intervention for SRM due to competing risks for mortality $(37,38,50,53)$. However, of the older patients managed with SRM, the proportion who were managed with AS is in the minority for most age groups, with approximately $10 \%$ of those aged 70-79 years on AS, rising to approximately $30 \%$ in $80-89$ year, and AS not chosen in the majority $(>70 \%)$ until the $90+$ age group (17). 
The mean age of AS patients for the studies in this review ranged from 63 to 81 years, with the average across studies of 70 years (Table 4). This is compared with the age range for all patients with SRM or those who had PI which was 60 to 79 years, with an average age across studies of 64 years. Three studies $(17,20,21,23)$, found that patients selected for AS were significantly older than those receiving PI, whereas age was not associated with use of AS in three other studies $(15,16,18)$. Of the studies with predefined recommendations for AS selection, only one [Haifa (25)] does not mention age as a factor.

\section{Risk of primary operative management}

Although age is the strongest predictor of risk of death from non-cancer related causes, within the context of risk from operative management, it is not clear whether elderly patients have worse outcomes from nephrectomy than younger patients. There is evidence that $\mathrm{PN}$ has a higher rate of postoperative complications than $\mathrm{RN}$ (54) which may increase the risk of surgery for elderly patients. But there is also evidence to suggest that older patients (more than 70 years $v s$. those under 70 years) have no significantly increased complication rate after robotic PN (55), and that older patients may still benefit from $\mathrm{PN}$ rather than $\mathrm{RN}$ despite a higher reported complication rate overall for PN (56). However, this evidence is from a select, much smaller population of elderly patients undergoing $\mathrm{PN}$ compared to the younger cohort. Age alone should not preclude a patient from the option of PN for a SRM but given the lowrisk overall of cancer-specific mortality from SRM for these patients, surgery may not be of overall benefit to the patient.

Two studies $(19,22)$ in this review included 'unfit for surgery' as a selection criteria for AS, of which, the criteria in one of these studies (22) states 'advanced age' as a reason a patient may not be a surgical candidate. It could be argued that patients who, at baseline, are not surgical candidates should be placed on 'watchful waiting' which is not the same management as AS. Watchful waiting can be used for patients who are not suitable for any active treatment, with no need for imaging surveillance, and intervention would be triggered by clinical symptoms with the aim of palliation. Active surveillance is a deferred treatment option, where delayed intervention is triggered by progression evident on surveillance, with a curative aim. It is therefore not clear whether some of the patients in these AS cohorts were, in reality, on watchful waiting rather than AS, and brings into question the value of having 'unfit' for surgery as a criterion for AS. This may also depend on the use of thermal ablation as a non-surgical option for DI, which may widen the selection criteria for management with AS.

\section{Renal function}

Aside from elderly patients, those with, or at risk of, progression to end stage renal failure after nephrectomy should receive special consideration when balancing risk of immediate versus delayed intervention. Only one study [Haifa (25)] in this review specifically mentioned risk factors for end-stage renal disease as an inclusion criteria for AS. No studies in Table 4 that compared baseline renal function between AS and PI groups reported a statistically significant difference. Radical or partial nephrectomy does reduce kidney function but usually not to a significant extent to lead to end stage renal failure, due to the ability of the kidney to hypertrophy and compensate for nephron loss $(57,58)$. Recovery of renal function to baseline has been shown in $45 \%$ of patients two years after radical nephrectomy (59).

Even for patients who go on to have CKD after surgery, the outcomes are better than for those with medically induced CKD, where there are often persistent underlying causes such as hypertension and diabetes (57). Therefore, the consideration of risk in these patients should not primarily be focused on the loss of nephrons versus the risk of cancer. For those patients with SRM and preexisting late-stage CKD, the competing mortality risk of cardiovascular disease, which is the main driver of mortality in late stage $\mathrm{CKD}$, is the more significant factor and must be balanced against any potential oncological benefit. As the estimated glomerular filtration rate decreases to $<30 \mathrm{~mL} / \mathrm{min} / 1.73 \mathrm{~m}^{2}$, death due to cardiovascular disease will outpace death due to malignancy by a factor of $2: 1(57)$.

\section{Clinician factors}

Reasons behind patient and clinician choice of AS versus primary intervention may also include clinician factors. This is important to understand how clinicians make decisions on patient selection for AS given that the current guidelines and evidence for practice of AS allows for clinicians to use their own interpretation of whether patients meet the inclusion criteria for comorbidity and suitability for surgery. A survey of urologists shows that $34 \%$ would choose PN or $\mathrm{RN}$ for a patient with SRM in their 80s, decreasing to $16 \%$ if the patient also had significant comorbidities (60). Of those urologists who chose $\mathrm{PN}$ or $\mathrm{RN}$ for these cases, they themselves were more likely to be $>65$ years, practicing 
in a non-academic setting, and without oncology fellowship training, and stated that they did not consider patient age one of the 3 most important considerations in their decision making process (60). These trends for uptake of AS for SRM may change with the more recent 2017 AUA guidelines and EAU guidelines making specific mention of recommendations for AS $(1,8)$.

\section{Role of renal mass biopsy}

The use of renal mass biopsy is low across the studies included in this review and suggests, in practice, RMB is not a routine diagnostic procedure before consideration of AS. Table 2 shows the rate of RMB for patients with SRM varies from $2.3 \%$ to $56 \%$. Even for patients enrolled in a clinical trial for AS [Canada RCC Consortium (22)] where every participant was offered RMB, only $56 \%$ had RMB. The role of RMB may be particularly useful for a select group of patients considering AS and is mentioned in several of the guidelines. The low uptake of RMB may also be related to traditional concerns regarding its safety, validity, non-diagnostic rate and risk of seeding, issues which have largely been shown to be not as significant as previously thought in a 2016 systematic review and meta-analysis (61). This showed across 57 studies and 5,228 patients who had RMB for renal tumours (of which 7 studies included SRM only), there was a median diagnostic rate of $92 \%$, with sensitivity of $99 \%$ and specificity of $99 \%$ for renal core biopsies. There was an overall complication rate of $8.1 \%$ of which, only three cases had a complication of Clavien-Dindo grade $\geq 2$ (61).

Of the studies included in Table 2, non-diagnostic rates for RMB in these SRM cohorts are reported to be between $19-33 \%$, with benign findings in $12-40 \%$ of cases, and malignancy found in $28-80 \%$ of cases $(20,22,31,52)$. Given that growth rate has been shown to be similar between benign and malignant lesions, and imaging is not reliable in distinguishing between pathologically aggressive and low-risk tumours, a finding of benign histology is likely to avoid overtreatment of SRM on AS and perhaps reduce the intensity of follow-up on AS (62). Even for a biopsy finding of oncocytoma, observation has been shown to be safe after mid-term follow up as a significant number of oncocytomas will show zero or minimal growth (63).

For those with a finding of malignant histology on RMB, given the slow growth rate, low metastatic potential, and competing risks for mortality in certain patients, AS is still a safe choice and can reduce overtreatment in malignant
SRM. In a prospective study of SRM with biopsy proven RCC on AS, the majority showed growth on AS (59\%) but $27 \%$ showed a decrease in the size and the rest remained unchanged in size (22).

RMB may also reveal malignant tumours with more favourable histology (such as low grade clear cell RCC, type 1 papillary and chromophobe types) versus more aggressive histology with high grade RCC, which may influence the decision for DI on AS (64). However, there has been found to only be a fair to good level of agreement between biopsy and surgical pathology for tumour subtype $(\kappa=0.68)$ and Fuhrman grade $(\kappa=0.34)(61)$. Using RMB to identify highgrade or sarcomatoid RCC with a worse prognosis before AS is not reliable and may still not impact overall survival (20).

Therefore, it is not clear whether the additional information gained from initial RMB benefits the management of AS patients in terms of avoiding overtreatment and triggering appropriate DI (65). In a prospective database of 118 patients, 51 patients had RMB, of which $35 \%$ were malignant, $37 \%$ benign and $28 \%$ non-diagnostic (26). For those who went onto to have DI, compared to those on AS who did not proceed to intervention, the rate of initial RMB was the same, as was the proportion of RMB which were malignant (26). The risk for DI was shown to be mainly influenced by growth rate and initial size of tumour rather than the results of initial renal biopsy (26). This mirrors the finding of reasons for delayed intervention from two AS studies included in this review and other studies, with patient and clinician choice being the major decider rather than progression on imaging $(4,20,30,31)$.

It may be that RMB is of greater utility after a period of AS if the tumour is showing significant growth. If the RMB reveals a benign or good prognosis malignant tumour this may sway the decision to continue AS rather than proceed to delayed intervention, especially in those patients with a high, rather than intermediate, risk of competing risk for mortality $(20,65)$. Initial RMB before AS has also been shown to be associated with greater psychological distress in the over 70 age group if the result of RMB shows malignant histology, which may be counterproductive to effective AS management given the overall low metastatic risk of malignant SRM (66).

\section{Surveillance strategy and indications for delayed intervention}

\section{Evidence for the growth rate of the SRM}

Surveillance strategy currently relies on measuring the growth kinetics of the SRM but this has been shown to 
be an unreliable prognosticator of metastatic potential. The evidence from retrospective and prospective data suggests that SRM show growth of approximately 0.10 to $0.30 \mathrm{~cm}$ per year, with $10-20 \%$ showing a zero growth rate $(4,20,22,31,52,67,68)$. Unhelpfully, the growth rate between malignant $v s$. benign SRM does not seem to differ, and initial tumour size is also not predictive of growth rate $(4,68)$. The rate of metastases of SRM on AS has been recorded to be between $0.4-2 \%(4,20)$. However, the proportion of SRM on AS having had a renal mass biopsy (RMB) is variable, and therefore there exists contamination of the active surveillance data with benign lesions. Where biopsy has been performed or subsequent pathology is available from delayed intervention there is still a significant number of benign lesions being treated, with $41-88 \%$ of treated SRM having been found to be malignant, $12-18 \%$ benign, and $19-33 \%$ were non-diagnostic $(4,20,22,31,52)$.

\section{Indications for delayed intervention}

Few guidelines specify an imaging surveillance protocol, although studies of AS cohorts have shown similarities in their use of surveillance imaging and thresholds for intervention. However, rates of DI seem variable despite similar definitions for progression based on tumour size and growth rate. Even for the patients on AS in the DISSRM registry, with predefined criteria for progression, over half of the patients who underwent delayed intervention had no evidence of clinical progression (31). However, it is worth noting that of the 21 AS patients who crossed over to DI in the DISSRM 2015 study, the rate of RCC on final histology was $67 \%$ in the patient preference group (with 2 high grade tumours), and $83 \%$ in the group meeting DI criteria (with 1 high grade tumour) (20). This further emphasizes that the current indications for DI could be improved. In the Smaldone et al. (2012) systematic review of retrospective AS cohorts for SRM, $45.4 \%(129 / 284)$ of SRM underwent delayed intervention at a mean of $30.5 \pm 21.8$ months (4). Of the $85 / 129$ reasons given for delayed intervention, patient preference was given in $57 \%$ of cases and tumour growth in $36 \%$. The masses which underwent delayed intervention also had a significantly higher linear growth rate of $0.38 v s .0 .24 \mathrm{~cm} /$ year, although initial tumour diameter was similar between continued surveillance and DI groups (4). Of the prospective cohort studies in this systematic review, triggers for DI included tumour size $>4 \mathrm{~cm}$, increasing tumour complexity on imaging, symptoms, infiltrative appearance, patient preference, and interval growth of more than $0.5 \mathrm{~cm}$ per year $(4,48)$.

The finding of a significant proportion of cases of DI being due to patient choice across the studies included in this review should prompt further investigation of how patients are counselled during AS. The potential for psychological distress and anxiety of patients should also be considered for patients on AS and as a factor for why patients may choose to stop AS. Although the DISSRM registry suggested no difference in the mental component score of the SF-12 questionnaire between the AS and PI groups, patients who have 'illness uncertainty' have been shown to have worse physical and psychosocial quality of life scores (32). Another study of patients under 70 years on AS found greater psychological distress for AS patients compared to surgery/ablation patients, and a biopsy-proven malignancy was also a risk factor for greater psychological distress on AS (66).

\section{Imaging surveillance protocols}

Specific imaging protocols for surveillance of a SRM on AS do not have a strong evidence base. The use of CT $v$ s. MRI $v s$. US is also a balance of radiation risk versus the ability to accurately measure maximal tumour diameter and characterise enhancement in Hounsfield units. US and MRI may be preferred to reduce radiation dose and in those with poor renal function, but CT is preferential for Hounsfield unit enhancement characterisation (69). Serial measurement of maximal tumour diameter should ideally be done with a consistent imaging modality regardless of which one is chosen. Although tumour volume should give a better indicator of growth, the low inter-observer variability and margin of error shown with measurement of maximal tumour diameter suggests this is still the preferred way to monitor tumour growth (69).

Increased use of RMB before and during AS may improve understanding of when best to trigger delayed intervention, as well as imaging biomarkers to indicate tumour progression. Currently, tumour size and growth kinetics are the mainstay of imaging surveillance, but with no standardised approach. This may contribute to a lesser degree of confidence for both the clinician and patient when considering AS for SRM.

\section{The future of AS for low-risk kidney cancer}

AS for low-risk kidney cancer has been adopted into international guideline recommendations, and population data from the USA shows large numbers of patients are being managed with AS. However, evidence is still needed on diagnosis and risk stratification of the SRM in order to understand the risk of progression for patients entering AS 
and the impact of surveillance strategies. Initial tumour size and tumour growth rate have been shown to be unreliable predictors of malignant and aggressive disease.

Further evidence on the management of SRM and AS surveillance is needed from prospective trials with standardised protocols for patient selection and follow-up, and standardised reporting of clinical outcomes. Further evidence is awaited from five registered trials are currently collecting data for AS management of SRM, three in North America and two in Europe (47). The European Active Surveillance of Renal Cell Carcinoma (EASE RCC) study (47) is currently open and aims to recruit 400 patients across Europe with SRM with the primary objective to assess the survival of patients who are diagnosed with incidental SRM managed with AS. Crucially, this trial requires histological confirmation with $\mathrm{RMB}$ and aims to identify the clinical and pathological factors of growth rate and progression for SRM. The increased use of RMB in AS trials should reduce the number of patients included who do not have histologically proven kidney cancer.

Research priorities identified as part of a renal cancer modified Delphi consensus statement for low-risk kidney cancer include: improving the diagnosis and characterisation of incidentally detected SRM; developing an evidence-based active surveillance protocol based on the natural history of the SRM and the impact of different imaging protocols; and developing effective immunohistochemical biomarkers for kidney biopsy samples that can provide better diagnostic and prognostic information (70).

\section{Characterisation of SRM using imaging}

In terms of improving diagnosis and characterisation of the SRM, current methods rely on cross-sectional imaging and use of RMB. Renal tumours are difficult to characterise given the high degree of heterogeneity of renal tumour biology. Cross-sectional imaging has utility in distinguishing cystic versus solid RCCs and angiomyolipomas (AML). Cystic RCCs show a more indolent course regardless of size and Bosniak category and, although lipid-poor AMLs may be hard to distinguish from clear cell RCC, the vast majority of sporadic AMLs also demonstrate a negligible growth rate (48). Further discrimination of histological subtype, between clear cell RCC, oncocytoma, papillary and chromophobe RCC using CT and MRI is limited and not reliable enough to impact on clinical decision making (69). Novel imaging methods being investigated include $99^{\mathrm{m}} \mathrm{Tc}$-sestamibi singlephoton emission CT (SPECT/CT) and prostate-specific membrane antigen-targeted (PSMA) PET/CT to differentiate benign oncocytomas from RCC which may further improve risk stratification of SRM for primary intervention versus AS (48). A study of preoperative imaging with $99^{\mathrm{m}} \mathrm{Tc}$-sestamibi SPECT/CT of $50 \mathrm{~T} 1$ renal masses showed that SPECT/ CT was able to currently identify $5 / 6$ of oncocytomas and $2 / 2$ hybrid oncocytic/chromophobe tumours with a sensitivity of $87.5 \%$ and specificity of $95.2 \%$ (71).

\section{Tissue biomarkers}

As the role of RMB increases for the diagnosis of SRM, development of more reliable and readily available immunohistochemical and molecular tissue biomarkers that may be applied to biopsy samples will help improve the diagnostic, predictive and prognostic utility of RMB (70). The aims of a tissue biomarker would be to aid histological diagnosis, detect markers associated with aggressive disease and be able to diagnose benign tumours (47). In particular, distinguishing between benign and malignant oncocytomas remains a challenge. Chromophobe RCC is histologically and morphologically similar to benign renal oncocytomas, and molecular biomarkers which can be applied to RMB are being developed to aid diagnosis. It has been found that oncocytomas have more extensive overall abnormal DNA methylation compared to chromophobe RCC. Using DNA methylation patterns, a molecular biomarker based on a signature of differentially methylated cystosinephosphate-guanine sites (CpGs) has been developed which can distinguish oncocytoma from chromophobe RCC with $85 \%$ to $96 \%$ accuracy $(46,72)$. Another novel method is 'optical biopsy' which uses optical coherence tomography via a needle probe to image a renal tumour, allowing the analysis of tissue specific optical properties. Cancer tissue has different light scattering properties to normal tissue and different renal cancer subtypes have been shown to have different optical properties with this technique (71).

\section{Conclusion}

Evidence for the safety and strategy of AS has grown with increasing data from retrospective, and more recently, long term data from large prospective AS cohorts. The 10 -year update from the DISSRM registry includes 437 AS patients, the largest prospective AS cohort to date. Further data is awaited from ongoing prospective trials of AS to gain better understanding of the natural history of AS, the survival of AS patients, the clinical and pathological factors affecting growth and progression of AS and the optimal AS surveillance strategy. This review 
shows that AS is being applied in a variety of centres worldwide and that key areas of patient selection criteria and surveillance strategy have large similarities. However, the rate of renal mass biopsy and of delayed intervention varies significantly between studies, suggesting the process of diagnosing malignant SRM and decision making whilst on AS are varying in practice. Increased confidence with diagnosis and management of SRM on AS will depend on answering the key research questions of diagnosing the high risk malignant SRM and understanding the factors for progression.

\section{Acknowledgments}

Funding: None.

\section{Footnote}

Provenance and Peer Review: This article was commissioned by the Guest Editors (Mieke Van Hemelrijck and Netty Kinsella) for the series "Expectant Management in Genitourinary Malignancies (Prostate, Bladder, Kidney)" published in Translational Andrology and Urology. The article has undergone external peer review.

Conflicts of Interest: The authors have completed the ICMJE uniform disclosure form (available at http:// dx.doi.org/10.21037/tau-20-1295). The series "Expectant Management in Genitourinary Malignancies (Prostate, Bladder, Kidney)" was commissioned by the editorial office without any funding or sponsorship. The authors have no other conflicts of interest to declare.

Ethical Statement: the authors are accountable for all aspects of the work in ensuring that questions related to the accuracy or integrity of any part of the work are appropriately investigated and resolved.

Open Access Statement: This is an Open Access article distributed in accordance with the Creative Commons Attribution-NonCommercial-NoDerivs 4.0 International License (CC BY-NC-ND 4.0), which permits the noncommercial replication and distribution of the article with the strict proviso that no changes or edits are made and the original work is properly cited (including links to both the formal publication through the relevant DOI and the license). See: https://creativecommons.org/licenses/by-nc-nd/4.0/.

\section{References}

1. Ljungberg B, Albiges L, Abu-Ghanem Y, et al. European Association of Urology Guidelines on Renal Cell Carcinoma: The 2019 Update. Eur Urol 2019;75:799-810.

2. Van Poppel H Van, Pozzo L Da, Albrecht W, et al. A Prospective, Randomised EORTC Intergroup Phase 3 Study Comparing the Oncologic Outcome of Elective Nephron-Sparing Surgery and Radical Nephrectomy for Low-Stage Renal Cell Carcinoma. Eur Urol 2011;59:543-52.

3. Kunath F, Schmidt S, Krabbe LM, et al. Partial nephrectomy versus radical nephrectomy for clinical localised renal masses. Cochrane Database Syst Rev 2017;5:CD012045.

4. Smaldone MC, Kutikov A, Egleston BL, et al. Small renal masses progressing to metastases under active surveillance. Cancer 2012;118:997-1006.

5. Ristau BT, Kutikov A, Uzzo RG, et al. Active Surveillance for Small Renal Masses: When Less is More. Eur Urol Focus 2016;2:660-8.

6. AUA. Urological Worldwide Society Database [Internet]. [cited 2020 May 1]. Available online: www.auanet.org/ education/international-societies

7. Ljungberg B, Albiges L, Bensalah K, et al. EAU Guidelines on Renal Cell Carcinoma. In European Association of Urology Guidelines Office; 2020.

8. Campbell S, Uzzo RG, Allaf ME, et al. Renal mass and localized renal cancer: AUA Guideline. 2017.

9. Escudier B, Porta C, Schmidinger M, et al. Renal cell carcinoma: ESMO Clinical Practice Guidelines for diagnosis, treatment and follow-up. Ann Oncol 2019;30:706-20.

10. Jewett MAS, Rendon R, Lacombe L, et al. Canadian guidelines for the management of small renal masses (SRM). Can Urol Assoc J 2015;9:160-3.

11. Fujioka T, Obara W, the Committee for Establishment of the Clinical Practice Guideline for the Management of Renal Cell Carcinoma and the Japanese Urological Association . Evidence-Based Clinical Practice Guideline for Renal Cell Carcinoma: The Japanese Urological Association 2011 update. Int J Urol 2012;19:496-503.

12. Dieguez A. Consenso Nacional Inter-Sociedades para el diagnóstico y tratamiento de las neoplasias renales parenquimatosas del adulto. Rev Argentina Radiol 2010;74:267-82.

13. Alghamdi A, Alkhateeb S, Alghamdi K, et al. Saudi Oncology Society and Saudi Urology Association 
combined clinical management guidelines for renal cell carcinoma. Urol Ann 2016;8:136-40.

14. de Cássio Zequi S, da Costa WH, Korkes F, et al. Renal cell cancer treatment: an expert panel recommendation from the Latin American cooperative group-genitourinary and the Latin American renal cancer group: focus on surgery. Ther Adv Urol 2019;11:1756287219872324.

15. Jacobs BL, Tan H-J, Montgomery JS, et al. Understanding criteria for surveillance of patients with a small renal mass. Urology 2012;79:1027-32.

16. Smaldone MC, Churukanti G, Simhan J, et al. Clinical Characteristics Associated With Treatment Type for Localized Renal Tumors: Implications for Practice Pattern Assessment. Urology 2013;81:269-75.

17. Doolittle J, Piotrowski J, Zuk K, et al. Evolving Trends for Selected Treatments of T1a Renal Cell Carcinoma. Urology 2019;132:136-42.

18. Lane BR, Abouassaly R, Gao T, et al. Active treatment of localized renal tumors may not impact overall survival in patients aged 75 years or older. Cancer 2010;116:3119-26.

19. Mason RJ, Abdolell M, Trottier G, et al. Growth kinetics of renal masses: analysis of a prospective cohort of patients undergoing active surveillance. Eur Urol 2011;59:863-7.

20. Pierorazio PM, Johnson MH, Ball MW, et al. Five-year Analysis of a Multi-institutional Prospective Clinical Trial of Delayed Intervention and Surveillance for Small Renal Masses: The DISSRM Registry. Eur Urol 2015;68:408-15.

21. Cheaib J, Alam R, Kassiri B, et al. Active Surveillance for Small Renal Masses is Safe and Non-Inferior: 10-Year Update from the DISSRM Registry. J Urol 2020;203:e917.

22. Jewett MAS, Mattar K, Basiuk J, et al. Active Surveillance of Small Renal Masses: Progression Patterns of Early Stage Kidney Cancer. Eur Urol 2011;60:39-44.

23. Patel N, Cranston D, Akhtar MZ, et al. Active surveillance of small renal masses offers short-term oncological efficacy equivalent to radical and partial nephrectomy. BJU Int 2012;110:1270-5.

24. Sullivan ME, Patel N, Brown S, et al. 608 - Active surveillance of small renal masses in an older population offers long-term oncological efficacy equivalent to partial or radical nephrectomy. Eur Urol Open Sci 2020;19:e947-8.

25. Bahouth Z, Halachmi S, Meyer G, et al. The Natural History and Predictors for Intervention in Patients with Small Renal Mass Undergoing Active Surveillance. Meng M V, editor. Adv Urol 2015;2015:692014.

26. Ambani SN, Morgan TM, Montgomery JS, et al. Predictors of Delayed Intervention for Patients on Active
Surveillance for Small Renal Masses: Does Renal Mass Biopsy Influence Our Decision? Urology 2016;98:88-96.

27. Park S-W, Lee SS, Lee DH, et al. Growth kinetics of small renal mass: Initial analysis of active surveillance registry. Investig Clin Urol 2017;58:429-33.

28. Paterson C, Yew-Fung C, Sweeney C, et al. Predictors of growth kinetics and outcomes in small renal masses (SRM $\leq 4 \mathrm{~cm}$ in size): Tayside Active Surveillance Cohort (TASC) Study. Eur J Surg Oncol 2017;43:1589-97.

29. Xing M, Kokabi N, Zhang D, et al. Comparative Effectiveness of Thermal Ablation, Surgical Resection, and Active Surveillance for T1a Renal Cell Carcinoma: A Surveillance, Epidemiology, and End Results (SEER)-Medicare-linked Population Study. Radiology 2018;288:81-90.

30. Petros FG, Venkatesan AM, Kaya D, et al. Conditional survival of patients with small renal masses undergoing active surveillance. BJU Int 2019;123:447-55.

31. Alam R, Patel HD, Osumah T, et al. Comparative effectiveness of management options for patients with small renal masses: a prospective cohort study. BJU Int 2019;123:42-50.

32. Semerjian A, Alam R, Patel H, et al. Quality of Life Scores in Patients with Small Renal Masses who have Undergone Renal Mass Biopsy Compared to those who Have Not: Analysis of the DISSRM Registry. J Urol 2017;197:e1135.

33. Uzosike AC, Patel HD, Alam R, et al. Growth Kinetics of Small Renal Masses on Active Surveillance: Variability and Results from the DISSRM Registry. J Urol 2018;199:641-8.

34. Danzig MR, Ghandour RA, Chang P, et al. Active Surveillance is Superior to Radical Nephrectomy and Equivalent to Partial Nephrectomy for Preserving Renal Function in Patients with Small Renal Masses: Results from the DISSRM Registry. J Urol 2015;194:903-9.

35. Alam R, Patel HD, Riffon MF, et al. Intermediate-term outcomes from the DISSRM registry: A prospective analysis of active surveillance in patients with small renal masses. J Clin Oncol 2017;35:430.

36. Metcalf MR, Cheaib JG, Biles MJ, et al. Outcomes of Active Surveillance for Young Patients with Small Renal Masses: Prospective Data from the DISSRM Registry. J Urol. doi: http://dx.doi.org/10.1097/ JU.0000000000001575.

37. Sun M, Becker A, Tian Z, et al. Management of Localized Kidney Cancer: Calculating Cancer-specific Mortality and Competing Risks of Death for Surgery and Nonsurgical Management. Eur Urol 2014;65:235-41. 
38. Kutikov A, Egleston BL, Wong YN, et al. Evaluating overall survival and competing risks of death in patients with localized renal cell carcinoma using a comprehensive nomogram. J Clin Oncol 2010;28:311-7.

39. Yang G, Villalta JD, Meng M V, et al. Evolving practice patterns for the management of small renal masses in the USA. BJU Int 2012;110:1156-61.

40. Huang WC, Pinhiero LC, Lowrance WT, et al. Surveillance for the Management of Small Renal Masses: Outcomes in a Population-Based Cohort. J Urol 2013;189:e483.

41. Russell DH, Wachtel MS, de Riese HW, et al. Implications on clinical management of the small renal mass in patients 80 years of age and older based on a retrospective review of the SEER database. Int Urol Nephrol 2015;47:1503-8.

42. Kim SP, Gross CP, Meropol N, et al. National treatment trends among older patients with T1-localized renal cell carcinoma. Urol Oncol 2017;35:113.e15-113.e21.

43. Tan WS, Trinh QD, Hayn MH, et al. Delayed nephrectomy has comparable long-term overall survival to immediate nephrectomy for c'T1a renal cell carcinoma: A population-based analysis. Urol Oncol 2020; 38:74.e1374.e20.

44. Cooperberg MR, Mallin K, Kane CJ, et al. Treatment Trends for Stage I Renal Cell Carcinoma. J Urol 2011;186:394-9.

45. Shah PH, Alom MA, Leibovich BC, et al. The Temporal Association of Robotic Surgical Diffusion with Overtreatment of the Small Renal Mass. J Urol 2018;200:981-8.

46. Brennan K, Metzner TJ, Kao C-S, et al. Development of a DNA Methylation-Based Diagnostic Signature to Distinguish Benign Oncocytoma From Renal Cell Carcinoma. JCO Precis Oncol 2020;(4):1141-51.

47. Mir MC, Capitanio U, Bertolo R, et al. Role of Active Surveillance for Localized Small Renal Masses. Eur Urol Oncol 2018;1:177-87.

48. Sanchez A, Feldman AS, Hakimi AA. Current Management of Small Renal Masses, Including Patient Selection, Renal Tumor Biopsy, Active Surveillance, and Thermal Ablation. J Clin Oncol 2018;36:3591-600.

49. Almassi N, Gill BC, Rini B, et al. Management of the small renal mass. Transl Androl Urol 2017;6:923-30.

50. Cheung DC, Finelli A. Active Surveillance in Small Renal Masses in the Elderly: A Literature Review. Eur Urol Focus 2017;3:340-51.

51. Pierorazio PM, Johnson MH, Patel HD, et al. Management of Renal Masses and Localized Renal
Cancer: Systematic Review and Meta-Analysis. J Urol 2016;196:989-99.

52. Kunkle DA, Egleston BL, Uzzo RG. Excise, Ablate or Observe: The Small Renal Mass Dilemma; A MetaAnalysis and Review. J Urol 2008;179:1227-33.

53. Hollingsworth JM, Miller DC, Daignault S, et al. Fiveyear survival after surgical treatment for kidney cancer. Cancer 2007;109:1763-8.

54. Mir MC, Derweesh I, Porpiglia F, et al. Partial Nephrectomy Versus Radical Nephrectomy for Clinical T1b and T2 Renal Tumors: A Systematic Review and Meta-analysis of Comparative Studies. Eur Urol 2017;71:606-17.

55. Sandberg JM, Krane LS, Hemal AK. A Nonrandomized Prospective Comparison of Robotic-assisted Partial Nephrectomy in the Elderly to a Younger Cohort: An Analysis of 339 Patients With Intermediate-term Followup. Urology 2014;84:838-43.

56. An JY, Ball MW, Gorin MA, et al. Partial vs Radical Nephrectomy for T1-T2 Renal Masses in the Elderly: Comparison of Complications, Renal Function, and Oncologic Outcomes. Urology 2017;100:151-7.

57. Chang EH, Tan H-J, Nielsen M. Management of small renal masses in patients with chronic kidney disease: Perspectives from a nephrologist. Urol Oncol 2020;38:533-6.

58. Shuch B, Hanley JM, Lai JC, et al. Adverse Health Outcomes Associated with Surgical Management of the Small Renal Mass. J Urol 2014;191:301-8.

59. Zabor EC, Furberg H, Lee B, et al. Long-Term Renal Function Recovery following Radical Nephrectomy for Kidney Cancer: Results from a Multicenter Confirmatory Study. J Urol 2018;199:921-6.

60. Millman AL, Pace KT, Ordon M, et al. Surgeon-specific factors affecting treatment decisions among Canadian urologists in the management of pT1a renal tumours. Can Urol Assoc J 2014;8:183-9.

61. Marconi L, Dabestani S, Lam TB, et al. Systematic Review and Meta-analysis of Diagnostic Accuracy of Percutaneous Renal Tumour Biopsy. Eur Urol 2016;69:660-73.

62. Richard PO, Jewett MAS, Finelli A. Re: Alexander Kutikov, Marc C. Smaldone, Robert G. Uzzo, Miki Haifler, Gennady Bratslavsky, Bradley C. Leibovich. Renal Mass Biopsy: Always, Sometimes, or Never? Eur Urol 2017;71:e45-6.

63. Richard PO, Jewett MAS, Bhatt JR, et al. Active Surveillance for Renal Neoplasms with Oncocytic Features is Safe. J Urol 2016;195:581-6. 
64. Richard PO, Jewett MAS, Bhatt JR, et al. Renal Tumor Biopsy for Small Renal Masses: A Single-center 13-year Experience. Eur Urol 2015;68:1007-13.

65. Kutikov A, Smaldone MC, Uzzo RG, et al. Renal Mass Biopsy: Always, Sometimes, or Never? Eur Urol 2016;70:403-6.

66. Goldberg H, Ajaj R, Cáceres JOH, et al. Psychological distress associated with active surveillance in patients younger than 70 with a small renal mass. Urol Oncol 2020;38:603.e17.

67. Volpe A, Panzarella T, Rendon RA, et al. The Natural History of Incidentally Detected Small Renal Masses. Cancer 2004;100:738-45.

68. McIntosh AG, Ristau BT, Ruth K, et al. Active

Cite this article as: Cui HW, Sullivan ME. Surveillance for low-risk kidney cancer: a narrative review of contemporary worldwide practices. Transl Androl Urol 2021;10(6):2762-2786. doi: $10.21037 /$ tau-20-1295
Surveillance for Localized Renal Masses: Tumor Growth, Delayed Intervention Rates, and >5-yr Clinical Outcomes. Eur Urol 2018;74:157-64.

69. Liaw CW, Winoker JS, Mehrazin R. Imaging Protocols for Active Surveillance in Renal Cell Carcinoma. Curr Urol Rep 2018;19:81.

70. Rossi SH, Blick C, Handforth C, et al. Essential Research Priorities in Renal Cancer: A Modified Delphi Consensus Statement. Eur Urol Focus 2020;6:991-8.

71. Haifler M, Kutikov A. Update on Renal Mass Biopsy. Curr Urol Rep 2017;18:28.

72. Chopra S, Liu J, Alemozaffar M, et al. Improving needle biopsy accuracy in small renal mass using tumor-specific DNA methylation markers. Oncotarget 2017;8:5439-48. 